\title{
Motion and collision of particles near DST black holes
}

\author{
P. A. González ${ }^{1, \mathrm{a}}$, Marco Olivares ${ }^{1, \mathrm{~b}}$, Ali Övgün ${ }^{2,3, \mathrm{c}}$, Joel Saavedra ${ }^{2, \mathrm{~d}}$, Yerko Vásquez ${ }^{4, \mathrm{e}}$ \\ ${ }^{1}$ Facultad de Ingeniería y Ciencias, Universidad Diego Portales, Avenida Ejército Libertador 441, Casilla 298-V, Santiago, Chile \\ ${ }^{2}$ Instituto de Física, Pontificia Universidad Católica de Valparaíso, Casilla 4950, Valparaíso, Chile \\ ${ }^{3}$ Physics Department, Arts and Sciences Faculty, Eastern Mediterranean University, via Mersin 10, Famagusta, North Cyprus, Turkey \\ ${ }^{4}$ Departamento de Física y Astronomía, Facultad de Ciencias, Universidad de La Serena, Avenida Cisternas 1200, La Serena, Chile
}

Received: 20 March 2019 / Accepted: 11 June 2019 / Published online: 20 June 2019

(C) The Author(s) 2019

\begin{abstract}
We consider the Deser-Sarioglu-Tekin (DST) black hole as a background, and we study the motion of massive particles in the case that we have a collision of two spinning particles in the vicinity of its horizon. New kinds of orbits are allowed for small deviations of General Relativity, but the behavior of the collision is similar to the one observed for General Relativity. Some observables like the bending of light and the perihelion precession are analyzed.
\end{abstract}

\section{Contents}

1 Introduction . . . . . . . . . . . 1

2 DST black holes . . . . . . . . . . . . 2

3 Geodesics in the equatorial plane ....... . . 3

3.1 Null geodesics . . . . . . . . . . . . . . . . 4

3.1 .1 Radial motion . . . . . . . . . . . . 4

3.1 .2 Angular motion . . . . . . . . . 4

3.1 .3 Bending of light . . . . . . . . . 5

3.2 Timelike geodesics . . . . . . . . . . 6

3.2.1 Radial geodesics . . . . . . . . . . . 7

3.2.2 Angular geodesics . . . . . . . . . . . 7

3.2.3 Perihelion precession . . . . . . . . . 8

4 Collisions of spinning particles near DST black holes 10

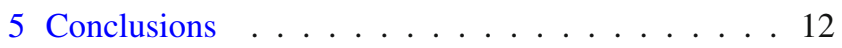

References . . . . . . . . . . . . . . . . . 13

\footnotetext{
a e-mail: pablo.gonzalez@udp.cl

b e-mail: marco.olivaresr@mail.udp.cl

c e-mail: ali.ovgun@pucv.cl

URL: http://www.aovgun.com

de-mail: joel.saavedra@ucv.cl

e e-mail: yvasquez@userena.cl
}

\section{Introduction}

The Deser-Sarioglu-Tekin (DST) action is characterized by the action of General Relativity (GR) with additional terms, i.e. non-polynomial terms of the Weyl tensor, that preserve the (first) derivative order of the GR equations in Schwarzschild gauge and provide non-Ricci flat extensions of GR. DST black holes are obtained using the Weyl technique for pure GR [2], which led to rather strange metrics [1]; however, GR can be recovered. Their thermodynamics was studied in [3].

The motion of particles in a spherically symmetric spacetime background has been of great interest. It is well known that all solar system observations, such as light deflection, the perihelion shift of planets, and the gravitational time-delay, are well described within Einstein's General Relativity. Also, observational data allow us to fix the parameters of alternative four-dimensional theories; see for instance [4,5]. In this regard, we address if new orbits appear and if the DST theory allows us to set the observables better than GR, for a small deviation from GR. The study of the motion of spinning tops (STOPs) in the framework of GR began with the work of Mathisson [6] and Papapetrou [7], which were again taken up by Tulczyjew [8], Taub [9], and Dixon [10]. The analysis of spinning particles moving around Schwarzschild black holes was first carried out by Corinaldesi and Papapetrou [11], who solved the Mathisson-Papapetrou (MP) equations, and later by Hojman [12], who solved the resulting equations derived from the Lagrangian formalism. An analytic treatment of the trajectories in general Schwarzschild-like spacetimes was carried out in [13], where it was found that a spinning test particle does not follow the geodesics due to its interaction with tidal forces.

On the other hand, it is well known that black holes can act as natural particle accelerators if in a collisional process of two particles near the degenerate horizon of an extreme 
Kerr black hole, one of the particles has a critical angular momentum by creating a large center of mass (CM) energy [15]. Nowadays, this process is known as the Bañados, Silk, and West (BSW) mechanism, which was found for the first time by Piran et al. [16-18]. The BSW mechanism can be extended to non-extremal black holes $[19,24]$ and to nonrotating charged black holes $[21,22]$. On top of that, it has been argued to be a universal property of rotating black holes [20]. The BSW mechanism has been studied for different black hole geometries [26-52]. Also, the formation of black holes through the BSW mechanism was investigated in [53]. It is worth mentioning that for the collision of STOPs in the equatorial plane of a Schwarzschild black hole, retrograde trajectories can experience significant accelerations, which generate divergent center-of-mass energies if the STOP collides with another particle moving in the same plane. However, in order to reach such a divergence, the trajectory of the STOP has to pass from timelike to spacelike [54]. In this regard, we address if the DST black hole can act as a particle accelerator.

The manuscript is organized as follows: In Sect. 2 we give a brief review of the DST black hole. Then we study and discuss the geodesics in the equatorial plane, and we analyze two observables, in particular the bending of light and the perihelion precession. Also, we find the values of the coupling parameter in order to set the observations in both tests, in Sect. 3. In Sect. 4 we study the collision of spinning particles, and we investigate the possibility that the DST black hole acts as a particle accelerator. Finally, our conclusions are in Sect. 5.

\section{DST black holes}

The action of Deser-Sarioglu-Tekin [1] corresponds to the Einstein action with the addition of non-polynomial terms, which in units of $\kappa=1$ is given by

$I=\frac{1}{2} \int d^{4} x \sqrt{-g}\left(R+\beta_{n}\left|\operatorname{tr} C^{n}\right|^{1 / n}\right)$,

where

$\operatorname{tr}^{n} \equiv C_{a b}{ }^{c d} C_{c d}{ }^{e f} \ldots C_{. .}{ }^{p q} C_{p q}{ }^{a b} \ldots$,

$n$ being the number of copies of the Weyl tensor $C$ and $\beta_{n}$ corresponding to the coupling constant. So, for $\mathrm{n}=2$ and by defining $\sigma=\beta_{2} / \sqrt{3}$, the above action can be written as

$I=\int_{0}^{\infty} \mathrm{d} r\left[(1-\sigma)\left(a r b^{\prime}+b\right)+3 \sigma a b\right]$, up to boundary terms. Here, primes denote radial derivatives. Thus, for $D=4$, the following metric is the solution of the field equations:

$\mathrm{d} s^{2}=-a(r) b^{2}(r) \mathrm{d} t^{2}+\frac{\mathrm{d} r^{2}}{a(r)}+r^{2} \mathrm{~d} \Omega_{2}$,

where

$a(r)=\frac{1-\sigma}{1-4 \sigma}+a_{1} r^{(4 \sigma-1) /(1-\sigma)}, \quad b(r)=b_{1} r^{3 \sigma /(\sigma-1)}$,

$a_{1}$ and $b_{1}$ are integration constants of which $b_{1}$ is removable by time rescaling. Note that for $\sigma=1$, there is no solution at all; for $\sigma=0$, GR is recovered, and for $\sigma=1 / 4, a(r)=$ $\ln \left(r / r_{0}\right)$ and $b(r)=1 / r$. All non-vanishing components of the mixed Weyl tensor are proportional to the single function $X$,

$$
\begin{aligned}
X(r, t) \equiv & \frac{1}{r^{2}}\left(2(a-1)-2 r a^{\prime}+r^{2} a^{\prime \prime}\right) \\
& +\frac{1}{r b}\left(3 r a^{\prime} b^{\prime}-2 a\left(b^{\prime}-r b^{\prime \prime}\right)\right)+\frac{1}{b} \partial_{t}\left(\frac{1}{a^{2} b} \partial_{t} a\right) .
\end{aligned}
$$

Also, any scalar of order $n$ in the Weyl tensor $C$ is proportional to $X^{n}$. Therefore

$\operatorname{tr} C^{n}=\left(-\frac{1}{3}\right)^{n}\left[2+(-2)^{2-n}\right] X^{n}$.

Note that the range $1 / 4<\sigma<1$ is excluded to retain the signature. However, for $a_{1}<0$, it is possible to recover the Schwarzschild metric. In the following we analyze the branch $a_{1}<0$, and we consider the functions $a(r)$ and $b(r)$ as

$a(r)=\frac{1-\sigma}{1-4 \sigma}-\left(\frac{r_{S}}{r}\right)^{\frac{1-4 \sigma}{1-\sigma}}$,
$b(r)=\left(\frac{r}{r_{S}}\right)^{\frac{3 \sigma}{\sigma-1}}$,

which are dimensionless, with $r_{S}$ being the Schwarzschild horizon coordinate. The black hole horizon is given by

$r_{+}\left(\sigma, r_{S}\right)=r_{S}\left(\frac{1-4 \sigma}{1-\sigma}\right)^{\frac{1-\sigma}{1-4 \sigma}}$

which depends on the parameter $\sigma$ and the Schwarzschild horizon. The event horizon, as a function of $\sigma$, has the maximum value $r_{+}^{(\max )} \approx 1.44 r_{S}$ when

$\sigma_{1}=-\frac{e-1}{4-e} \approx-1.34$

Also, when $\sigma \rightarrow \pm \infty, r_{+} \rightarrow \sqrt{2} r_{S}$; see Fig. 1. 


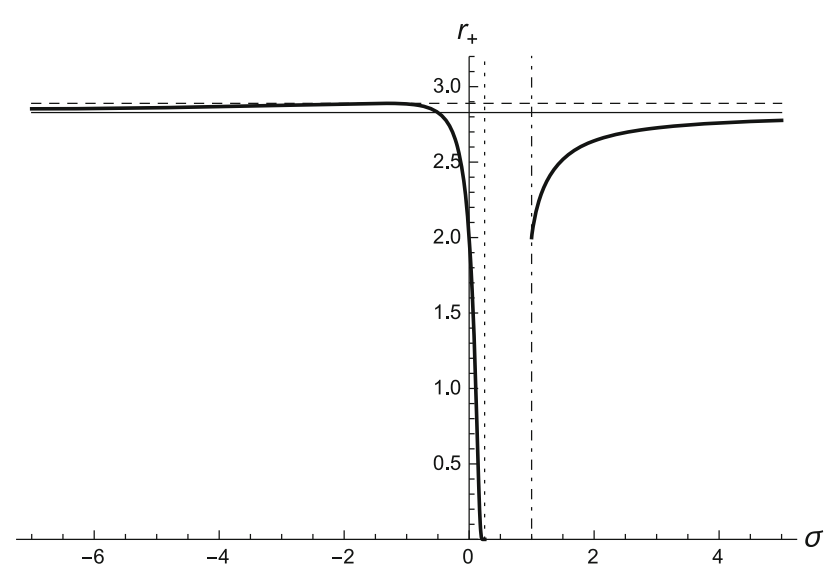

Fig. 1 The behavior of the DST horizon as a function of $\sigma$ with $r_{S}=2$

It is worth mentioning that the mass of the black hole solution could be computed following the usual Abbott-DeserTekin (ADT) formulation of conserved charges [55-58] or employing the off-shell ADT current which generalizes the usual ADT method [59]. In the on-shell ADT formalism the metric $g_{\mu \nu}$ is split into a background metric $\bar{g}_{\mu \nu}$ plus a perturbation $h_{\mu \nu}$; that is, $g_{\mu \nu}=\bar{g}_{\mu \nu}+h_{\mu \nu}$. Also, for the construction of a conserved current it is necessary to linearize the field equations, which are obtained by varying the action (1) with respect to the metric and can be written in a generic form as

$\mathcal{E}_{\mu \nu}=\kappa T_{\mu \nu}$

We consider the background metric

$\mathrm{d} \bar{s}^{2}=-\frac{1-\sigma}{1-4 \sigma} r^{-\frac{6 \sigma}{1-\sigma}} \mathrm{d} t^{2}+\frac{1-4 \sigma}{1-\sigma} \mathrm{d} r^{2}+r^{2} \mathrm{~d} \Omega_{2}$,

where $a_{1}=0$ and $b_{1}$ has been removed by time rescaling from Eqs. (4) and (5). In the following, all the barred quantities refer to the background metric $\bar{g}_{\mu \nu}$. Thus, it is possible to find the linearized tensor $\mathcal{E}_{\mu \nu}^{(1)}(h)$ [60]. Therefore, given a background Killing vector $\bar{\xi}^{\mu}$ and using the linearized tensor $\mathcal{E}_{\mu \nu}^{(1)}(h)$, a partially conserved current can be constructed:

$j^{\mu}=\sqrt{-\bar{g}} \bar{\xi}_{\nu} \mathcal{E}^{(1) \mu \nu}, \partial_{\mu} j^{\mu}=0$

Now, integrating $\partial_{\mu} j^{\mu}$ on the background manifold $\overline{\mathcal{M}}$ and using the Stokes theorem, the conserved charge as an integral over a spatial hypersurface $\bar{\Sigma}$ is given by

$Q(\bar{\xi})=\int_{\bar{\Sigma}} d^{3} y \sqrt{\bar{\gamma}} \bar{n}_{\mu} \bar{\xi}_{\nu} \mathcal{E}^{(1) \mu \nu}$

where $\bar{\gamma}$ is the induced metric on the boundary of $\overline{\mathcal{M}}$ and $\bar{n}^{\mu}$ is a unit normal vector to the boundary $\partial \overline{\mathcal{M}}$. Addition- ally, $\bar{\xi}_{\nu} \mathcal{E}^{(1) v \mu}$ can be written as the divergence of a tensor, $\bar{\xi}_{\nu} \mathcal{E}^{(1) \nu \mu}=\bar{\nabla}_{\nu} \mathcal{F}^{\mu \nu}(\bar{\xi})$, where $\mathcal{F}^{\mu \nu}$ is antisymmetric, and using the Stokes theorem again the following expression can be obtained:

$Q(\bar{\xi})=\int_{\partial \bar{\Sigma}} d^{2} z \sqrt{\bar{\gamma}^{(\partial \bar{\Sigma})}} \bar{\epsilon}_{\mu \nu} \mathcal{F}^{\mu \nu}(\bar{\xi})$,

where $\partial \bar{\Sigma}$ is the boundary of $\bar{\Sigma}, \bar{\gamma}^{(\partial \bar{\Sigma})}$ is the induced metric on $\partial \bar{\Sigma}$ and the antisymmetric binomial vector is defined by $\bar{\epsilon}_{\mu \nu}=\frac{1}{2}\left(\bar{n}_{\mu} \bar{\sigma}_{\nu}-\bar{n}_{\nu} \bar{\sigma}_{\mu}\right)$, and $\bar{\sigma}^{\mu}$ is the outward unit normal on $\partial \bar{\Sigma}$. With this expression and considering the background timelike Killing vector $\bar{\xi}=\partial_{t}$, one can compute the mass of the solution, which should be related to the parameter $a_{1}$; for $\sigma \rightarrow 0$, we expect to recover the mass of the Schwarzschild black hole. On the other hand, in the off-shell ADT formalism [59], an off-shell Noether current $J^{\mu}$ can be defined, which generalizes the on-shell current (14), and the off-shell ADT potential $\mathcal{F}^{\mu \nu}$ can be defined as $J^{\mu}=\bar{\nabla}_{\nu} \mathcal{F}^{\mu \nu}$. Using this potential, and following a similar procedure to above, an expression for the conserved charges can be obtained as an integral at spatial infinity on the boundary of the spacelike hypersurface. In addition, the expression obtained can be evaluated more easily using an important relation between the off-shell Noether potential and the ADT potential. We refer the reader to [59] for details.

\section{Geodesics in the equatorial plane}

The Lagrangian associated with the metric (4) is

$2 \mathcal{L}=-a b^{2} \dot{t}^{2}+\frac{\dot{r}^{2}}{a}+r^{2} \dot{\theta}^{2}+r^{2} \sin ^{2} \theta \dot{\phi}^{2}$,

where $\dot{q}=\mathrm{d} q / \mathrm{d} \lambda$, and $\lambda$ is an affine parameter along the geodesic that we choose as the proper time $\tau$ for particles. Since the Lagrangian (17) is independent of the coordinates $(t, \phi)$, their conjugate momenta $\left(\Pi_{t}, \Pi_{\phi}\right)$ are conserved. The equations of motion can be obtained from

$\dot{\Pi}_{q}-\frac{\partial \mathcal{L}}{\partial q}=0$

which yield

$$
\begin{aligned}
\dot{\Pi}_{t}= & 0, \quad \dot{\Pi}_{r}=-\frac{\dot{t}^{2}}{2} \frac{\mathrm{d}\left(a b^{2}\right)}{\mathrm{d} r}+\frac{\dot{r}^{2}}{2} \frac{\mathrm{d}\left(a^{-1}\right)}{\mathrm{d} r} \\
& +r \dot{\theta}^{2}+r \sin ^{2} \theta \dot{\phi}^{2}, \\
\dot{\Pi}_{\theta}= & r^{2} \sin \theta \cos \theta \dot{\phi}^{2}, \quad \text { and } \dot{\Pi}_{\phi}=0,
\end{aligned}
$$

where $\Pi_{q}=\partial \mathcal{L} / \partial \dot{q}$ are the conjugate momenta to the coordinate $q$, in particular

$$
\Pi_{t}=-a b^{2} \dot{t}, \quad \Pi_{r}=\frac{\dot{r}}{a},
$$


$\Pi_{\theta}=\dot{r}^{2} \dot{\theta}$, and $\Pi_{\phi}=r^{2} \sin ^{2} \theta \dot{\phi}$.

So, by considering the motion of neutral particles on the equatorial plane, $\theta=\pi / 2$ and $\dot{\theta}=0$, we obtain

$\Pi_{t}=-a b^{2} \dot{t} \equiv-E, \quad \Pi_{r}=\frac{\dot{r}}{a}, \quad \Pi_{\phi}=r^{2} \dot{\phi} \equiv L$,

where $E$ and $L_{\phi}$ are dimensionless integration constants. Now, by using Eqs. (8) and (11), the Lagrangian can be rewritten in the following form:

$2 \mathcal{L} \equiv-m=-\frac{E^{2}}{a b^{2}}+\frac{\dot{r}^{2}}{a}+\frac{L^{2}}{r^{2}}$

So, by normalization, we shall consider that $m=1$ for massive particles and $m=0$ for photons. We solve the above equation for $\dot{r}^{2}$ in order to obtain the radial equation, which allows us to characterize the possible movements of test particles without an explicit solution of the equation of motion in the invariant plane, and we obtain

$$
\begin{aligned}
& \left(\frac{\mathrm{d} r}{\mathrm{~d} \lambda}\right)^{2}=\frac{1}{b^{2}}\left[E^{2}-V(r)\right], \\
& \left(\frac{\mathrm{d} r}{\mathrm{~d} t}\right)^{2}=\frac{a^{2} b^{2}}{E^{2}}\left[E^{2}-V(r)\right], \\
& \left(\frac{\mathrm{d} r}{\mathrm{~d} \phi}\right)^{2}=\frac{r^{4}}{L^{2} b^{2}}\left[E^{2}-V(r)\right],
\end{aligned}
$$

where $V(r)$ is the effective potential given by

$V(r)=a b^{2}\left(m+\frac{L^{2}}{r^{2}}\right)$.

\subsection{Null geodesics}

\subsubsection{Radial motion}

The radial motion corresponds to a trajectory with null angular momentum (or zero impact parameter). In this case, the photons are destined to escape to infinity or fall into the black hole because the effective potential is $V(r)=0$. Also, Eqs. (26) and (27) reduce to

$\pm \frac{\mathrm{d} r}{\mathrm{~d} \lambda}=\frac{E}{b}$

and

$\pm \frac{\mathrm{d} r}{\mathrm{~d} t}=a b$

respectively. The sign $+(-)$ corresponds to photons that escape (falling) from the event horizon. Now, choosing the initial conditions for the photons as $r=\rho_{0}$ when $t=\lambda=0$, Eq. (29) yields

$\lambda(r)= \pm \frac{r_{S}}{E}\left(\frac{1-\sigma}{1-4 \sigma}\right)\left[\left(\frac{r}{r_{s}}\right)^{\frac{1-4 \sigma}{1-\sigma}}-\left(\frac{\rho_{0}}{r_{S}}\right)^{\frac{1-4 \sigma}{1-\sigma}}\right]$.

Thus, the photons arrive at the event horizon for a finite $\lambda$ parameter, which can be observed in Fig. 2. Notice that photons plunge to the horizon with the same energy. The photon in the neighborhood of a DST black hole with positive $\sigma$ $(0<\sigma<1 / 4)$ reaches a point (outside the horizon) in a smaller affine parameter than a photon in the neighborhood of a DST black hole with a negative $\sigma$.

On the other hand, performing the change of variables $x=\left(r / r_{+}\right)^{v+1}$, and integrating Eq. (30) leads to

$t(r)= \pm r_{s}\left(\frac{r_{+}}{r_{S}}\right)^{1-v}\left(B[x ; z+1,0]-B\left[x_{0} ; z+1,0\right]\right)$

where $B[x ; \bar{\alpha}, \bar{\beta}]$ corresponds to the Beta function, $z=(1-$ $v) /(1+v)$ and $(1-\sigma) /(1-4 \sigma)=1 /(1+v)$. Notice that the solution for the coordinate time does not depend on the energy of the photon. In Fig. 3, we can observe that the photons in the coordinate time do not cross the horizon of the DST black holes.

\subsubsection{Angular motion}

The effective potential for photons and their trajectories are plotted in Fig. 4. The effective potential presents a maximum value, which corresponds to an unstable circular orbit with a radius given by

$r_{U}=r_{S}\left(\frac{3(1-4 \sigma)}{2(1-\sigma)(1+2 \sigma)}\right)^{\frac{1-\sigma}{1-4 \sigma}}$

The classical result for Schwarzschild's spacetime $\left(r_{U}=\right.$ $3 M$ ) is obtained when $\sigma=0$, and $r_{S}=2 M$. It is worth mentioning that there is a maximum in the potential only for $-1 / 2<\sigma<1 / 4$. On the other hand, the potential shows a different behavior for $\sigma \leq-1 / 2$. It is worth mentioning that stable circular orbits are not allowed for photons.

Now, based on the impact parameter $\beta \equiv L / E$, we give a brief qualitative description of the allowed angular motions for the photons plotted in Fig. 4. First, we can observe a capture zone, if $0<\beta<\beta_{c}$, where $\beta_{c}=L / E_{c}$ with $E_{c}^{2}=$ $V\left(r_{U}\right)$, where the photons fall to the horizon, depending on the initial conditions; their cross section, $\bar{\sigma}$, in this geometry is [70]

$\bar{\sigma}=\pi \beta_{c}^{2}$ 
Fig. 2 The behavior of the affine parameter $\lambda$ as a function of $r$, for different values of $\sigma$, with $E=1, \rho_{0}=6, r_{S}=2$. Vertical lines correspond to the event horizon for different $\sigma$ values
Fig. 3 The behavior of the coordinate time $t$ as a function of $r$, for different values of $\sigma$, with $\rho_{0}=6$, and $r_{S}=2$.

Vertical lines correspond to the event horizon for different $\sigma$ values
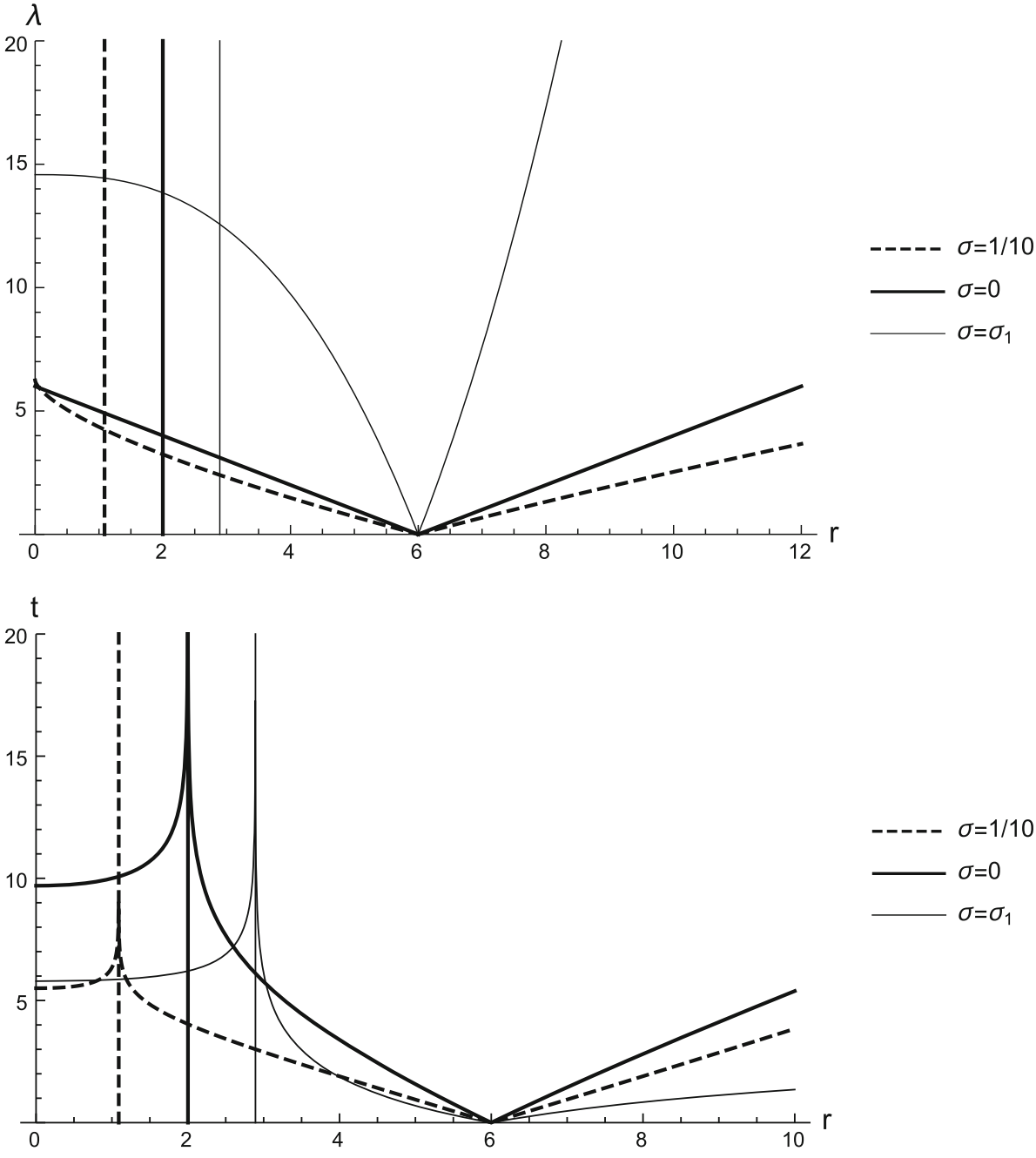

have a return point and then plunge into the black hole; see Fig. 5.

\subsubsection{Bending of light}

In this section we will follow the procedure established in Ref. [65]. So, Eq. (27) for photons is

$\left(\frac{\mathrm{d} r}{\mathrm{~d} \phi}\right)^{2}=\frac{r_{S}^{2 v}}{\beta^{2}} r^{4-2 v}-\mu r^{2}+r_{S}^{1 / \mu} r^{1-v}$

As a result it is found to be the same as the one in the Schwarzschild case [71], when $\sigma=0$. Also, the coordinate period is given by

$T_{t}=2 \pi \beta_{c}$.

Finally, there is a deflection zone, if $\beta_{c}<\beta_{d}<\infty$, where the photons come from infinity to a distance $r=r_{d}$ (which is the solution of the equation $V\left(r_{d}\right)=E^{2}$ ) and then return to infinity.

As we mentioned, the potential shows a different behavior for $\sigma \leq-1 / 2$. For $\sigma=-1 / 2$, the potential tends to $L^{2} / 2$, for $\sigma<-1 / 2$ the potential shows that all trajectories allowed where $\beta$ is the impact parameter, $v=3 \sigma /(\sigma-1)$, and $\mu=$ $(1-\sigma) /(1-4 \sigma)$. By using the change of variables $r=1 / u$, the above equation can be written as

$\left(\frac{\mathrm{d} u}{\mathrm{~d} \phi}\right)^{2}=\frac{r_{S}^{2 v}}{\beta^{2}} u^{2 v}-\mu u^{2}+r_{S}^{1 / \mu} u^{3+v}$

Notice that for $\sigma=0$, the above equation is reduced to the classical equation of Schwarzschild for the motion of photons 

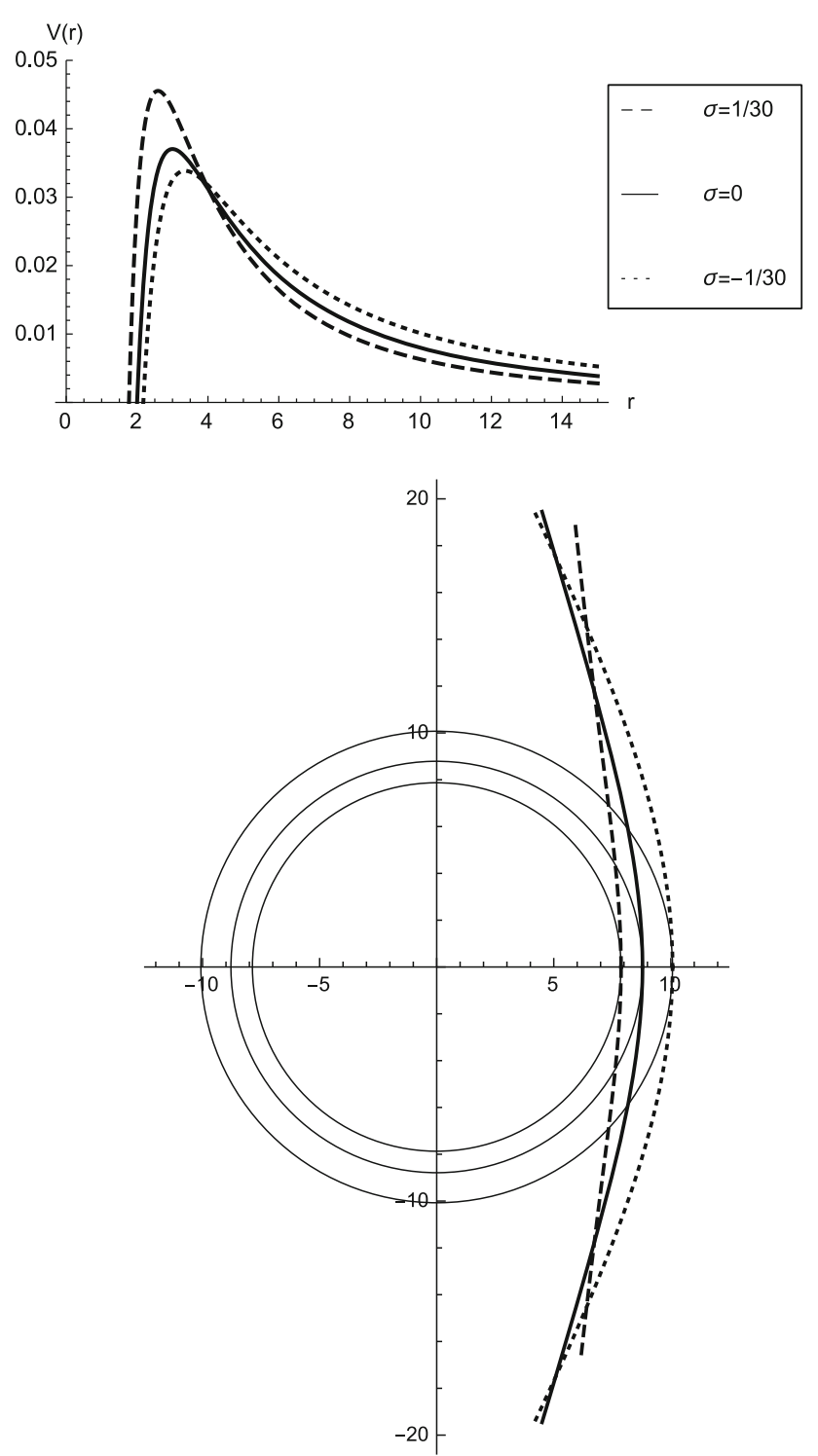

Fig. 4 The top panel shows the behavior of the effective potential for photons $\mathrm{V}(\mathrm{r})$ as a function of $r$, for different values of $\sigma$, with $r_{S}=2$ and $L=1$. The bottom panel shows the trajectories for photons with $E^{2}=0.01$, and different values of $\sigma$

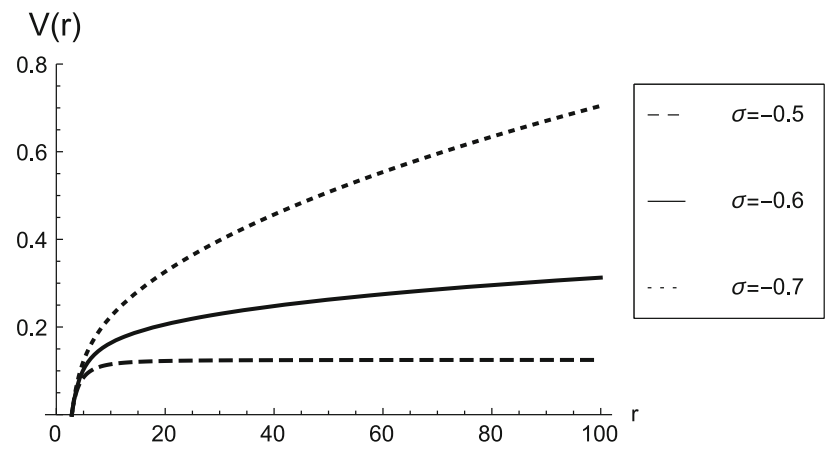

Fig. 5 The behavior of the effective potential for photons $\mathrm{V}(\mathrm{r})$ as a function of $r$, for $\sigma \leq-1 / 2$, with $r_{S}=2$ and $L=1$ for $r_{S}=2 M$,

$$
\left(\frac{\mathrm{d} u}{\mathrm{~d} \phi}\right)^{2}=\frac{1}{\beta^{2}}-u^{2}+2 M u^{3} .
$$

So, the derivative of Eq. (38) with respect $\phi$ yields

$u^{\prime \prime}+\mu u=\frac{r_{S}^{1 / \mu}}{2}(3+v) u^{2+v}+\frac{v r_{S}^{2 v}}{\beta^{2}} u^{2 v-1}$

where' denotes the derivative with respect to $\phi$. Now, neglecting the last term, we obtain

$u=\frac{1}{\beta} \sin (\sqrt{\mu} \phi)+\frac{\epsilon}{\beta^{2+v} 2^{1+v / 2} \mu}\left(1+\frac{2+v}{6} \cos (2 \sqrt{\mu} \phi)\right)$,

where $\epsilon=r_{S}^{1 / \mu}(3+v) / 2$. For large $r$ (small $\left.u\right), \phi$ is small, and we may take $\sin (\sqrt{\mu} \phi) \approx \sqrt{\mu} \phi$ and $\cos (2 \sqrt{\mu} \phi) \approx 1$. In the limit $u \rightarrow 0, \phi$ approaches $\phi_{\infty}$, with

$\phi_{\infty}=-\frac{(3+v)(8+v) r_{S}^{1 / \mu}}{6 \mu^{3 / 2} \beta^{1+v} 2^{2+v / 2}}$.

Therefore, for the DST black holes, the deflection of light $\hat{\alpha}$ is equal to $2\left|\phi_{\infty}\right|$ and yields

$\hat{\alpha}=\frac{(3+v)(8+v)}{3 \mu^{3 / 2} 2^{2+v / 2}}\left(\frac{r_{s}}{\beta}\right)^{1+v}$.

Notice that for $\sigma=0$ and $r_{S}=2 M$, we recovered the classical result of GR; that is, $\hat{\alpha}=4 M / \beta$. The first observational value of the deflection of light was measured by Eddington and Dyson in the solar eclipse of March 29, 1919. For the Sobral expedition this value is $\hat{\alpha}_{\text {Obs. }}=1.98 \pm 0.16^{\prime \prime}$, and it was $\hat{\alpha}_{\text {Obs. }}=1.61 \pm 0.40^{\prime \prime}$ for the Principe expedition [65]. Nowadays, the parameterized post-Newtonian (PPN) formalism introduces the phenomenological parameter $\gamma$, which characterizes the contribution of space curvature to gravitational deflection. In this formalism the deflection angle is $\hat{\alpha}=0.5(1+\gamma) 1.7426$, and currently $\gamma=0.9998 \pm 0.0004$ [72]. So, $\hat{\alpha}=1.74277^{\prime \prime}$ for $\gamma=0.9998+0.0004$ and $\hat{\alpha}=1.74208^{\prime \prime}$ for $\gamma=0.9998-0.0004$.

It is worth mentioning that there is a discrepancy between the theoretical value predicted by GR and the observational value. So, by attributing this discrepancy to small deviations of Schwarzschild's spacetime, we can attribute such a discrepancy to $\sigma$. Therefore, $-8.97241 \times 10^{-6}<\sigma<$ $3.41708 \times 10^{-6}$, in order to match with the observational results.

\subsection{Timelike geodesics}

In this section, we will study the motion of massive particles and the perihelion precession. In the following, we fix $m=1$. 


\subsubsection{Radial geodesics}

The effective potential for particles $(L=0)$ is plotted in Fig. 6. Notice that, for $\sigma=0$, there are two kind of trajectories. One of them is the bounded trajectory $(E<1)$, which has a return point and plunges into the horizon. The other one is the unbounded trajectory ( $E \geq 1$ ), which can escape to infinity or plunge into the black hole. For $\sigma<0$, we observe that the allowed trajectories are bounded. Interestingly, for $0<\sigma<1 / 4$, the potential has a maximum value $V\left(r_{u}\right) \equiv E_{u}^{2}$, at the unstable equilibrium point $\left(r_{u}\right)$, which is not present in GR $(\sigma=0)$, and which can be obtained from the derivative of Eq. (28) with respect to $r$. This unstable equilibrium point is given by

$r_{u}=r_{S}\left(\frac{(1-4 \sigma)(1+2 \sigma)}{6 \sigma(1-\sigma)}\right)^{\frac{1-\sigma}{1-4 \sigma}}$.

Also, for this range of values of $\sigma$, there are three kinds of trajectories. The first are the critical trajectories, of the first and second kind, which are allowed for particles with $E=E_{u}$. The trajectories of the first kind are characterized by particles that are asymptotically incoming from infinity to the unstable equilibrium point. The trajectories of the second kind are characterized by particles that are asymptotically incoming from a distance, $r<r_{u}$, to the unstable equilibrium point. For particles with $E>E_{u}$, the trajectories are unbounded, and for $E<E_{u}$, we observe that a frontal scattering is allowed, which is characterized by particles that are incoming from infinity to a radial distance of closest approach, and then go back to infinity. The frontal scattering for charged particles was studied in Ref. [66].

\subsubsection{Angular geodesics}

The effective potential for particles with positive angular momentum is plotted in Fig. 7. Note that for the cases that have been analyzed the effective potential has extremal values that correspond to circular orbits (c.o.). The maximum value corresponds to the unstable circular orbit, whereas the minimum value corresponds to the innermost stable circular orbit (ISCO). The circular orbits correspond to the roots $\left(r_{\text {c.o. }}\right)$ of the following equation:

$$
\begin{aligned}
V^{\prime}= & \frac{2 \nu \mu}{r_{S}^{2 v}} r^{2 v-1}-r_{S}^{1-v}(v-1) r^{\nu-2} \\
& +\frac{2 \nu \mu L^{2}}{r_{S}^{2 v}}(2 v-2) r^{2 v-3}-r_{S}^{1-v} L^{2}(v-3) r^{\nu-4}=0,
\end{aligned}
$$

which does not have an analytical solution. However, for $\sigma=0$ the above equation yields

$$
V^{\prime}=\frac{r_{S}}{r^{2}}-\frac{2 L^{2}}{r^{3}}+\frac{3 r_{S} L^{2}}{r^{4}}=0
$$

whose roots correspond to the Schwarzschild case $(r=$ $\left.L^{2}\left(1 \pm \sqrt{1-3 r_{S}^{2} / L^{2}}\right) / r_{S}\right)$, where the $(+)$ sign is for the ISCO and the $(-)$ sign corresponds to the unstable circular orbit. On the other hand, the periods for one complete revolution of these circular orbits, measured in proper time and coordinate time, are

$T_{\tau}=2 \pi\left[\frac{(2-2 v) \mu r_{S}^{-2 v} r_{c . o .}^{3}-(3-v) r_{S}^{1-v} r_{c . o .}^{2-v}}{(1-v) r_{S}^{1-v} r_{c . o}^{-v}+2 v \mu r_{S}^{-2 v} r_{c . o .}}\right]^{1 / 2}$

$T_{t}=2 \pi\left[\frac{2 r_{c . o}^{3-2 v}}{(1-v) r_{S}^{1-v} r_{c . o}^{-v}+2 v \mu r_{S}^{-2 v} r_{c . o .}}\right]^{1 / 2}$,

for $\sigma=0$; the periods are given by

$$
\begin{aligned}
T_{\tau} & =2 \pi\left[\frac{2 r_{\text {c.o. }}^{3}}{r_{S}}-3 r_{\text {c.o. }}^{2}\right]^{1 / 2}, \\
T_{t} & =2 \pi \sqrt{\frac{2 r_{\text {c.o. }}^{3}}{r_{S}}},
\end{aligned}
$$

which matches with the periods for Schwarzschild spacetime. Now, expanding the effective potential around $r=r_{\text {ISCO }}$, we can write

$$
\begin{aligned}
V(r)= & V\left(r_{\mathrm{ISCO}}\right)+V^{\prime}\left(r_{\mathrm{ISCO}}\right)\left(r-r_{\mathrm{ISCO}}\right) \\
& +\frac{1}{2} V^{\prime \prime}\left(r_{\mathrm{ISCO}}\right)\left(r-r_{\mathrm{ISCO}}\right)^{2}+\cdots,
\end{aligned}
$$

where' means derivative with respect to the radial coordinate. Obviously, in these orbits $V^{\prime}\left(r_{\mathrm{ISCO}}\right)=0$. So, by defining the smaller coordinate $x=r-r_{\mathrm{ISCO}}$, together with the epicycle frequency $\kappa^{2} \equiv V^{\prime \prime}\left(r_{\mathrm{ISCO}}\right)$ [67], we can rewrite the above equation as $V(x) \approx E_{\mathrm{ISCO}}^{2}+\kappa^{2} x^{2}$, where $E_{\mathrm{ISCO}}^{2}$ is the energy of the particle in the stable circular orbit. Also, it is easy to see that test particles satisfy the harmonic equation of motion, $\ddot{x}=-\kappa^{2} x$. Therefore, in our case, the epicycle frequency is given by

$\kappa^{2}=\kappa_{0}\left(\kappa_{1}-\kappa_{2}\right)$,

where

$\kappa_{0}=\frac{1}{r_{\mathrm{ISCO}}^{3}}\left[(1-v) r_{S}^{1-v} r_{\mathrm{ISCO}}^{-v}+2 v \mu r_{S}^{-2 v} r_{\mathrm{ISCO}}\right]$,

$\kappa_{1}=\frac{2(1-v)(3-2 v) \mu r_{S}^{-2 v} r_{\text {ISCO }}^{1+2 v}-(3-v)(4-v) r_{S}^{1-v} r_{\text {ISCO }}^{v}}{(2-2 v) \mu r_{S}^{-2 v} r_{\text {ISCO }}-(3-v) r_{S}^{1-v} r_{\text {ISCO }}^{-v}}$,

$\kappa_{2}=\frac{(1-v)(2-v) r_{S}^{1-v} r_{\text {ISCO }}^{v}-2 v(2 v-1) \mu r_{S}^{-2 v} r_{\text {ISCO }}^{1+2 v}}{(1-v) r_{S}^{1-v} r_{\text {ISCO }}^{-v}+2 v \mu r_{S}^{-2 v} r_{\text {ISCO }}}$.

Notice that when $\sigma=0, \kappa \rightarrow \kappa_{S}$, where $\kappa_{S}$ is the epicycle frequency in the Schwarzschild case, given by

$\kappa_{S}^{2}=\frac{r_{S}}{r_{\text {ISCO }}^{3}}\left[\frac{r_{\text {ISCO }}-3 r_{S}}{r_{\text {ISCO }}-3 r_{S} / 2}\right]$. 
Fig. 6 The behavior of the effective potential for particles $\mathrm{V}(\mathrm{r})$ as a function of $r$, for different values of $\sigma, r_{S}=2$ and $L=0$. For $\sigma=0$ the potential $V(\infty)=1$, for $\sigma<0$ the potential $V(\infty)=\infty$, and for $0<\sigma<1 / 4$ the potential $V(\infty)=0$

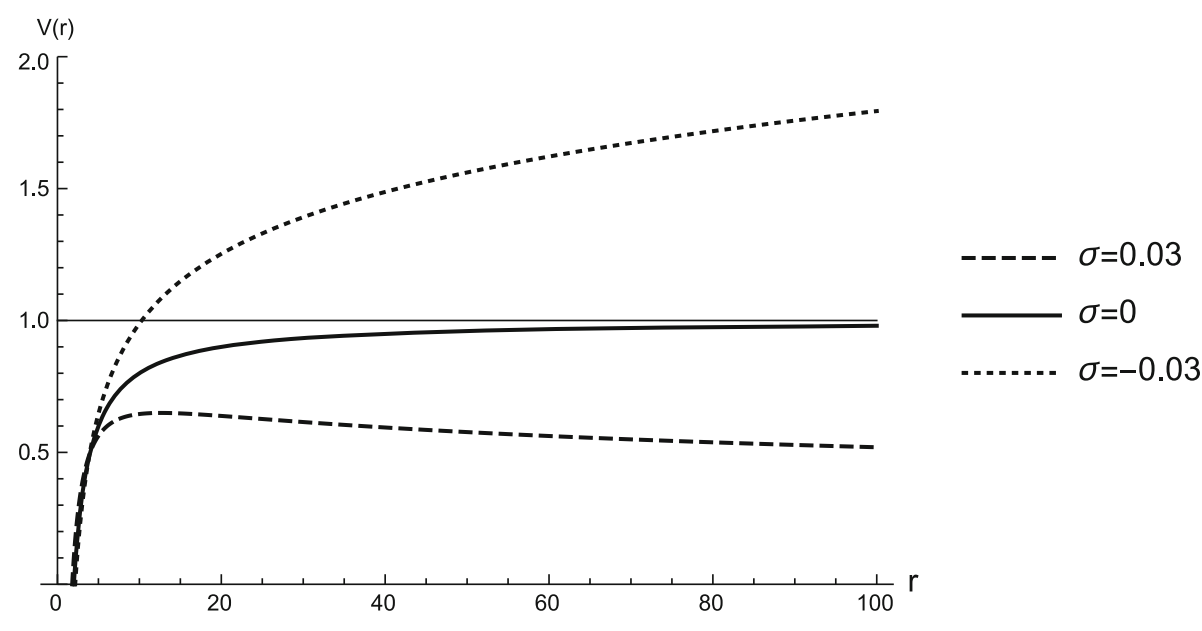

$\frac{\mathrm{d} A}{\mathrm{~d} t}=\frac{1}{2} \mathcal{R}^{2} \frac{\mathrm{d} \phi}{\mathrm{d} t}$,

Also, there are bound orbits like planetary orbits, as in GR [73]; for instance see the left panel of Fig. 8 for a positive $\sigma$. Moreover, for $\sigma<0$, all the trajectories are bounded due to $V(\infty)=\infty$. It is worth mentioning that these kinds of orbits have the same behavior as the timelike orbits for a Schwarzschild AdS black hole [68]. Interestingly, for $0<$ $\sigma<1 / 4$, the spacetime allows for two unstable circular orbits and one ISCO. In the right panel of Fig. 8, we show the scattering of neutral particles with $E<1$, which are not present in Schwarzschild spacetime, which can be a repulsive scattering or an attractive scattering.

\subsubsection{Perihelion precession}

The previous analysis of the effective potential for particles showed that there are planetary orbits which allow us to study the perihelion precession. So, we follow the treatment performed by Cornbleet [69], which allows us to derive the formula for the advancement of the perihelia of planetary orbits. The starting point is considering the line element in unperturbed Lorentz coordinates,

$\mathrm{d} s^{2}=-\mathrm{d} t^{2}+\mathrm{d} r^{2}+r^{2}\left(\mathrm{~d} \theta^{2}+\sin ^{2} \theta \mathrm{d} \phi^{2}\right)$,

together with the line element (4). So, considering only the radial and time coordinates in the binomial approximation, and $b(r) \approx 1$, when $\sigma \rightarrow 0$ in the Newtonian limit. So, the transformation gives

$$
\begin{aligned}
\mathrm{d} \tilde{t} & \approx \sqrt{\mu}\left(1-\frac{1}{2 \mu}\left(\frac{r_{S}}{r}\right)^{\frac{1}{\mu}}\right) \mathrm{d} t, \\
\mathrm{~d} \tilde{r} & \approx \frac{1}{\sqrt{\mu}}\left(1+\frac{1}{2 \mu}\left(\frac{r_{S}}{r}\right)^{\frac{1}{\mu}}\right) \mathrm{d} r .
\end{aligned}
$$

We will consider two elliptical orbits, one the classical Kepler orbit in $(r, t)$ space and a DST black hole orbit in $(\tilde{r}, \tilde{t})$ space. Then in the Lorentz space $d A=\int_{0}^{\mathcal{R}} r \mathrm{~d} r \mathrm{~d} \phi=\mathcal{R}^{2} \mathrm{~d} \phi / 2$, and hence which corresponds to Kepler's second law. For the DST black hole case we have

$\mathrm{d} \tilde{A}=\int_{0}^{\mathcal{R}} r \mathrm{~d} \tilde{r} \mathrm{~d} \phi$,

where $d \tilde{r}$ is given by Eq. (59). So, we can write (61) as

$$
\begin{aligned}
\mathrm{d} \tilde{A} & =\frac{1}{\sqrt{\mu}} \int_{0}^{\mathcal{R}} r\left(1+\frac{1}{2 \mu}\left(\frac{r_{S}}{r}\right)^{1+v}\right) \mathrm{d} r \mathrm{~d} \phi \\
& =\frac{1}{2 \sqrt{\mu}}\left(\mathcal{R}^{2}+\frac{r_{S}^{1+v} \mathcal{R}^{1-v}}{\mu(1-v)}\right) \mathrm{d} \phi .
\end{aligned}
$$

Therefore, applying the binomial approximation we obtain

$$
\begin{aligned}
\frac{\mathrm{d} \tilde{A}}{\mathrm{~d} \tilde{t}} & =\frac{1}{2 \sqrt{\mu}}\left(\mathcal{R}^{2}+\frac{r_{S}^{1+v} \mathcal{R}^{1-v}}{\mu(1-v)}\right) \frac{\mathrm{d} \phi}{\mathrm{d} \tilde{t}} \\
& \approx \frac{\mathcal{R}^{2}}{2 \mu}\left(1+\frac{(3-v)}{2 \mu(1-v)}\left(\frac{r_{S}}{\mathcal{R}}\right)^{1+v}\right) \frac{\mathrm{d} \phi}{\mathrm{d} t} .
\end{aligned}
$$

So, we use this increase to improve the elemental angle from $\mathrm{d} \phi$ to $\mathrm{d} \tilde{\phi}$. Then for a single orbit

$$
\int_{0}^{\Delta \tilde{\phi}} \mathrm{d} \tilde{\phi}=\int_{0}^{\Delta \phi=2 \pi} \frac{1}{\mu}\left(1+\frac{(3-v)}{2 \mu(1-v)}\left(\frac{r_{S}}{\mathcal{R}}\right)^{1+v}\right) \mathrm{d} \phi .
$$

Now, the polar form of an ellipse is given by

$$
\mathcal{R}=\frac{l}{1+\epsilon \cos \phi},
$$

where $\epsilon$ is the eccentricity and $l$ is the semi-latus rectum; by plugging Eq. (65) into Eq. (64), we obtain

$$
\Delta \tilde{\phi}=\frac{1}{\mu}\left(2 \pi+\frac{(3-v) r_{S}^{1+\nu}}{2 \mu(1-v)} \int_{0}^{2 \pi}\left(\frac{1+\epsilon \cos \phi}{l}\right)^{(1+v)} d \phi\right),
$$


Fig. 7 The behavior of the effective potential for particles $\mathrm{V}(\mathrm{r})$ as a function of $r$, for different values of $\sigma$, with $r_{S}=2$ and $L=4$. For $\sigma=0$ the potential $V(\infty)=1$, for $\sigma<0$ the potential $V(\infty)=\infty$, and for $0<\sigma<1 / 4$ the potential $V(\infty)=0$

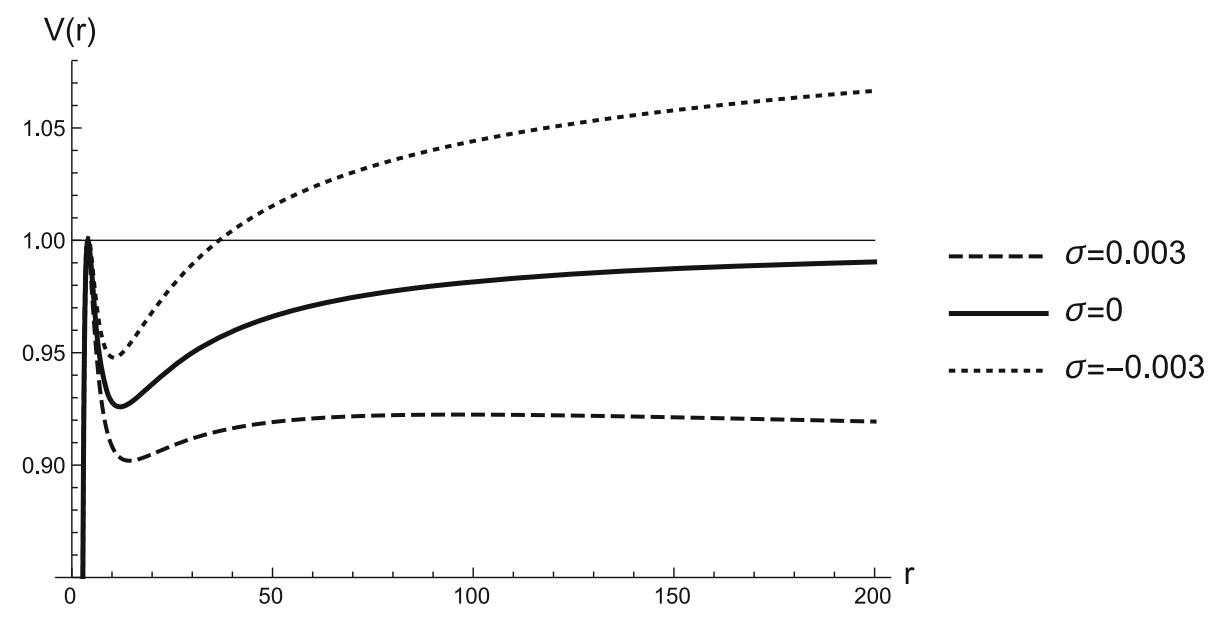

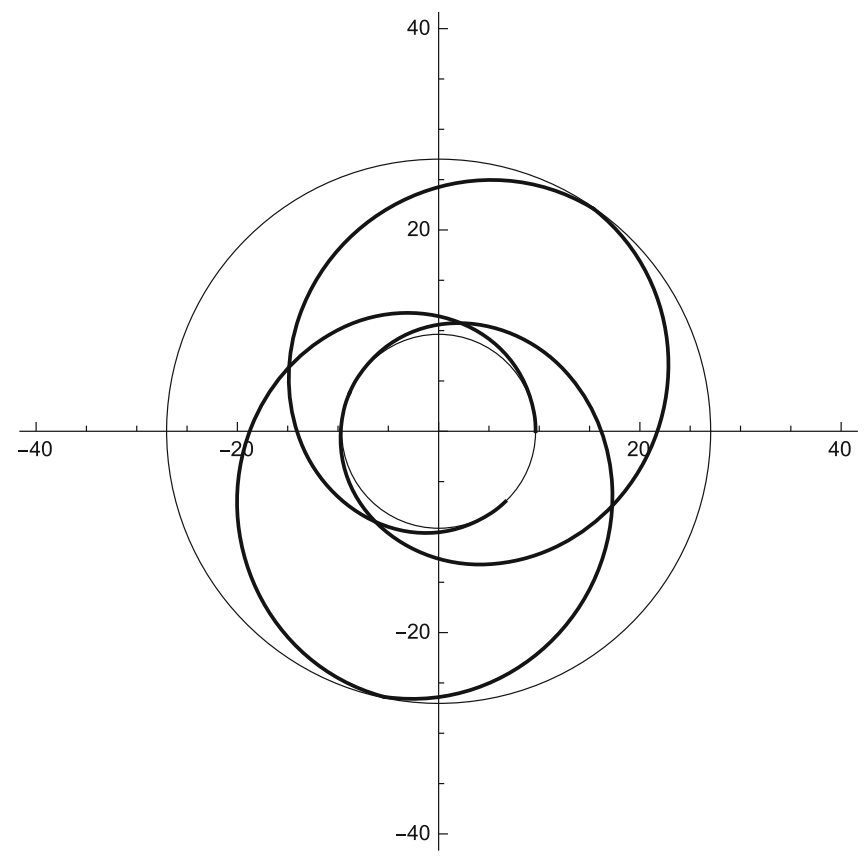

Fig. 8 Trajectories of particles with angular momentum $L=4$ in the background of DST black hole with $r_{S}=2$ and $\sigma=0.003$. Left panel: for bounded orbits like planetary orbit with energy $E^{2}=0.91$. Right panel: for the scattering of neutral particles with different values

which at the first order yields

$\Delta \tilde{\phi} \approx \frac{1}{\mu}\left(2 \pi+\frac{\pi(3-v)}{\mu(1-v)}\left(\frac{r_{S}}{l}\right)^{1+v}\right)$.

Notice that, for $\sigma=0$ and $r_{S}=2 M$, we recover the classical result of GR. It is worth mentioning that there is a discrepancy between the observational value of the precession of perihelion for Mercury, $\Delta \tilde{\phi}_{\text {Obs. }}=5599.74(\operatorname{arcsec} / J u l i a n-$ century) and the total $\Delta \tilde{\phi}=\Delta \phi_{\mathrm{eq}}+\Delta \phi_{\mathrm{pl}}+\Delta \phi_{\mathrm{obl}}=$ 5603.24 ( $\operatorname{arcsec} / J u l i a n-c e n t u r y)$, where the term $\Delta \phi_{\text {eq }}$ is caused by the general precession in longitude, the term $\Delta \phi_{\mathrm{pl}}$ is caused by the gravitational tugs of the other planets, and the

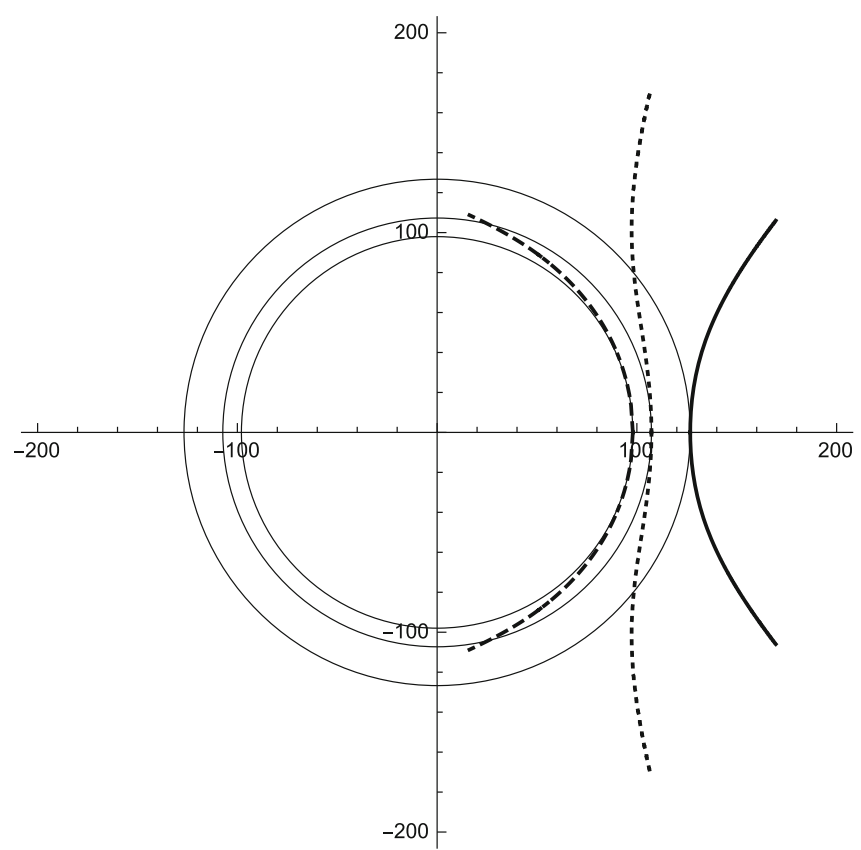

of energy, the continuous line corresponds to $E^{2}=0.922$ (repulsive scattering), the dashed line corresponds to $E^{2}=0.92247$ (attractive scattering), and the dotted line corresponds to $E^{2}=0.9224$

term $\Delta \phi_{\text {obl }}$ is caused by the oblateness of the Sun [4], which is possibly attributed to a DST theory with $\sigma=1.244 * 10^{-9}$.

The parameter $\sigma$ turns out to be a small parameter in matching the observed data. Thus, it is possible to write the DST metric as a series expansion in $\sigma$; at the first order in $\sigma$ the metric is

$$
\begin{aligned}
\mathrm{d} s^{2}= & {\left[\left(1-\frac{r_{S}}{r}\right)+\left(3-6 \ln \left[\frac{r}{r_{S}}\right]+3 \frac{r_{s}}{r} \ln \left(\frac{r}{r_{S}}\right) \sigma\right] d t^{2}\right.} \\
& +\frac{\mathrm{d} r^{2}}{\left(1-\frac{r_{S}}{r}\right)+\left(3+\frac{3 r_{S} \ln \left[\frac{r_{S}}{r}\right]}{r}\right) \sigma}+r^{2} \mathrm{~d} \Omega_{2}
\end{aligned}
$$


By comparing this metric with the Schwarzschild metric, we can observe that the components $g_{t t}$ and $g_{r r}$ contain the Schwarzschild component plus a correction introduced by the DST theory.

\section{Collisions of spinning particles near DST black holes}

The equations of motion derived from the Lagrangian theory for a spinning particle are given by $[12,14]$

$$
\begin{aligned}
\frac{D P^{\mu}}{D \tau} & =-\frac{1}{2} R_{\nu \alpha \beta}^{\mu} u^{\nu} S^{\alpha \beta}, \\
\frac{D S^{\mu \nu}}{D \tau} & =S^{\mu \beta} \sigma_{\beta}^{v}-\sigma^{\mu \beta} S_{\beta}^{v} \\
& =P^{\mu} u^{v}-u^{\mu} P^{v},
\end{aligned}
$$

where $D / D \tau \equiv u^{\mu} \nabla_{\mu}$ is the covariant derivative along the velocity vector $u^{\mu}, \tau$ is an affine parameter, $P^{\mu}$ is the canonical momentum, $R_{\nu \alpha \beta}^{\mu}$ is the Riemann tensor, $u^{\mu}=\mathrm{d} X^{\mu} / \mathrm{d} \tau$ is the tangent vector to the trajectory, $S^{\mu \nu}$ is the canonical spin tensor, and $\sigma^{\mu \nu}$ is the angular velocity. Spinning test particles in cosmological and static spherically symmetric spacetimes have been studied in [13]. Also, the collision of spinning particles near a Schwarzschild black hole was analyzed in [54]. In the following, we will consider the motion of spinning particles in the equatorial plane; that is, $\theta=\pi / 2$ and $P^{\theta}=0$. The modulus of the antisymmetric spin tensor and the mass of the particle are conserved quantities and are given, respectively, by

$$
\begin{gathered}
S^{2}=\frac{1}{2} S_{\mu \nu} S^{\mu \nu}, \\
m^{2}=-P_{\mu} P^{\mu} .
\end{gathered}
$$

Other constants of motion are given by

$$
Q_{\xi}=P^{\mu} \xi_{\mu}-\frac{1}{2} S^{\mu v} \nabla_{\nu} \xi_{\mu}
$$

where $\xi^{\mu}$ is a Killing vector of the spacetime. The conserved quantity associated with the Killing vector $\partial_{t}$ corresponds to the energy of the top and is found to be

$$
E=a(r) b^{2}(r) P^{t}-\frac{1}{2} S^{t r}\left(a^{\prime}(r) b^{2}(r)+2 a(r) b(r) b^{\prime}(r)\right) .
$$

Meanwhile, the conserved quantity associated with $\partial_{\phi}$ corresponds to the angular momentum of the top,

$$
J=r^{2} P^{\phi}+r S^{r \phi} .
$$

Also, the Tulczyjew constraint restricts the spin tensor to generate rotations only:

$S^{\mu v} P_{\nu}=0$.

Thus, using the above equations, we find that the nonvanishing components of momentum are given by

$$
\begin{aligned}
\frac{P^{t}}{m} & =\frac{e r^{3}-\frac{j s r^{2}}{2 b}\left(a^{\prime} b^{2}+2 a b b^{\prime}\right)}{a b^{2} \Sigma}, \\
\frac{P^{\phi}}{m} & =\frac{j r-\frac{e s r}{b}}{\Sigma},
\end{aligned}
$$

and

$$
\frac{P^{r}}{m}= \pm \sqrt{a^{2} b^{2}\left(\frac{P^{t}}{m}\right)^{2}-a r^{2}\left(\frac{P^{\phi}}{m}\right)^{2}-a},
$$

where $e=E / m$ is the specific energy, $j=J / m$ is the total angular momentum per unit mass and $s= \pm S / m$ is the spin per unit mass. While a positive value of the spin means that the spin is parallel to the total angular momentum, a negative value means that the spin is antiparallel to the total angular momentum. Also, $\Sigma$ is given by

$$
\begin{aligned}
\Sigma & =r^{3}-\frac{s^{2} r^{2}}{2}\left(a^{\prime}+2 a \frac{b^{\prime}}{b}\right) \\
& =r^{3}-\frac{s^{2}}{2}\left(r \Delta^{\prime}-\left(1-2 r \frac{b^{\prime}}{b}\right) \Delta\right),
\end{aligned}
$$

where we have defined $\Delta=r a(r)$. Thus, by considering that the center of mass energy is given by

$E_{\mathrm{CM}}^{2}=-g_{\mu \nu}\left(P_{1}^{\mu}+P_{2}^{\mu}\right)\left(P_{1}^{\nu}+P_{2}^{\nu}\right)=m_{1}^{2}+m_{2}^{2}-2 g_{\mu \nu} P_{1}^{\mu} P_{2}^{\nu}$,

then the collisional energy for two spinning particles with the same mass $m=m_{1}=m_{2}$ in the background of the DST black hole yields

$$
\begin{aligned}
E_{\mathrm{CM}}^{2}= & \frac{2 m^{2}}{\Delta \Sigma_{1} \Sigma_{2}}\left(\frac{r}{b^{2}}\left(e_{1} r^{3}-\frac{j_{1} s_{1}}{2} r^{2}\left(a^{\prime} b+2 a b^{\prime}\right)\right)\right. \\
& \left(e_{2} r^{3}-\frac{j_{2} s_{2}}{2} r^{2}\left(a^{\prime} b+2 a b^{\prime}\right)\right) \\
& +\Delta\left(\Sigma_{1} \Sigma_{2}-r^{4}\left(j_{1}-e_{1} s_{1} / b\right)\left(j_{2}-e_{2} s_{2} / b\right)\right) \\
& -\frac{1}{b^{2}} \sqrt{r\left(e_{1} r^{3}-\frac{j_{1} s_{1}}{2} r^{2}\left(a^{\prime} b+2 a b^{\prime}\right)\right)^{2}-\Delta b^{2}\left(\Sigma_{1}^{2}+r^{4}\left(j_{1}-e_{1} s_{1} / b\right)^{2}\right)} \\
& \times \sqrt{\left.r\left(e_{2} r^{3}-\frac{j_{2} s_{2}}{2} r^{2}\left(a^{\prime} b+2 a b^{\prime}\right)\right)^{2}-\Delta b^{2}\left(\Sigma_{2}^{2}+r^{4}\left(j_{2}-e_{2} s_{2} / b\right)^{2}\right)\right)} .
\end{aligned}
$$

Possibles divergences can arise when the denominator of the above equation is zero, i.e., at the horizon radius $\Delta=0$ and at a spin-related radius $\Sigma_{i}=0$. In the first case, it can be demonstrated that the CM energy is finite at the horizon. In 
Fig. 9 The behavior of $E_{C M}^{2}$ as a function of $r$, for $a_{1}=-2$,

$b_{1}=1, s_{1}=1, s_{2}=2.5$, $e_{1}=e_{2}=j_{1}=j_{2}=1$, $m_{1}=m_{2}=1$ and $\sigma=0.1$. The red line corresponds to the event horizon radius $r_{H} \approx 1.54$. $E_{C M}^{2}$ diverges at the spin-related radius

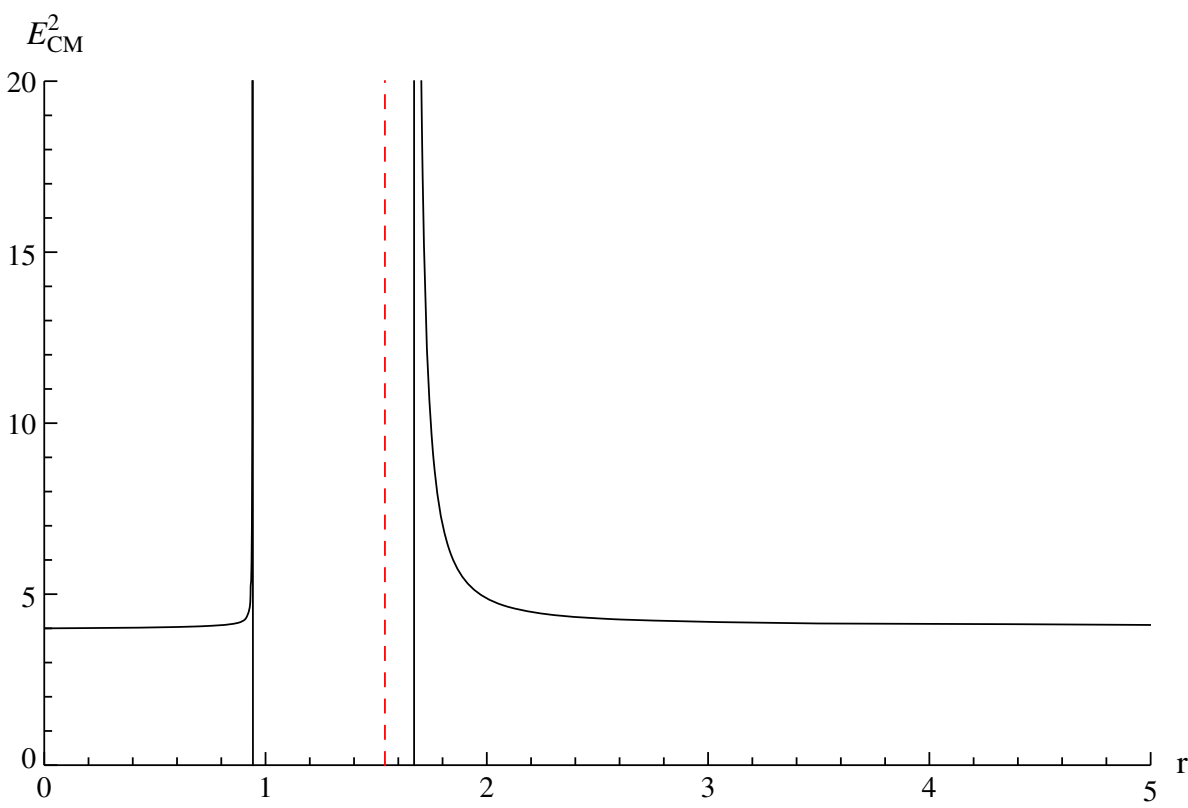

fact, setting $s_{i}=0$ for simplicity in the above expression, with $i=1,2$, for $E_{\mathrm{CM}}^{2}$ yields the finite value

$\lim _{r \rightarrow r_{H}} \frac{E_{\mathrm{CM}}^{2}}{m^{2}}=\frac{\left(e_{2} j_{1}-e_{1} j_{2}\right)^{2}+\left(e_{1}+e_{2}\right)^{2} r_{H}^{2}}{e_{1} e_{2} r_{H}^{2}}$.

In the case $s_{i} \neq 0$, it is more difficult to find an analytic expression like the above expression; however, it can be shown that $E_{\mathrm{CM}}^{2}$ also is finite when $\Delta \rightarrow 0$. For instance, in Fig. 9 we can see that the CM energy does not diverge at the horizon for some particular values of the parameters. On the other hand, when $\Sigma_{i} \rightarrow 0, E_{\mathrm{CM}}^{2}$ diverges, indicating an infinite $\mathrm{CM}$ energy at the spin-related radius. This behavior is shown in the same Fig. 9 for some values of the parameters, which shows that the divergence of the CM energy occurs outside the event horizon. However, it must be analyzed if the spinning particles can reach the divergence radius. In [54] it was shown that, for the Schwarzschild black hole, some particles with retrograde orbits in principle can reach the divergence radius before reaching the horizon.

Now, rewriting $P^{r}$ as

$$
\left(\frac{P^{r}}{m}\right)^{2}=\frac{1-\frac{a s^{2}}{r^{2}}}{b^{2}\left(1-\frac{s^{2}}{2 r}\left(a^{\prime}+2 a \frac{b^{\prime}}{b}\right)\right)^{2}}\left(e-V_{+}\right)\left(e-V_{-}\right),
$$

where the effective potentials $V_{ \pm}$are defined as

$$
V_{ \pm}=\left(1-\frac{a s^{2}}{r^{2}}\right)^{-1}\left(\left(a^{\prime} b r^{2}+2 a b^{\prime} r^{2}-2 a b r\right) \frac{j s}{2 r^{3}}\right.
$$

$$
\left.\pm \sqrt{a\left(1+\frac{j^{2}}{r^{2}}-\frac{a s^{2}}{r^{2}}\right)\left(1-\frac{s^{2}}{2 r}\left(a^{\prime}+2 a \frac{b^{\prime}}{b}\right)\right)^{2}}\right) .
$$

Notice that for the case $1-a s^{2} / r^{2}>0, e$ must be bigger than $V_{+}$or smaller than $V_{-}$for a real value of $P^{r}$. Therefore, from Eq. (69) and Eq. (70) we obtain the following set of momentum equations:

$$
\begin{aligned}
\dot{P}^{t} & +\left(\frac{a^{\prime}}{2 a}+\frac{b^{\prime}}{b}\right) P^{t} \dot{r}+\left(\frac{a^{\prime}}{2 a}+\frac{b^{\prime}}{b}\right) P^{r} \dot{t} \\
& =\left(\frac{3 a^{\prime} b^{\prime}}{2 a b}+\frac{a^{\prime \prime}}{2 a}+\frac{b^{\prime \prime}}{b}\right) S^{t r} \dot{r}+\left(a^{\prime}+2 a \frac{b^{\prime}}{b}\right) \frac{r}{2} S^{t \phi} \dot{\phi} \\
\dot{P}^{r} & +\frac{1}{2} a b^{2}\left(a^{\prime}+2 a \frac{b^{\prime}}{b}\right) P^{t} \dot{t}-\frac{a^{\prime}}{2 a} P^{r} \dot{r}-r a P^{\phi} \dot{\phi} \\
& =a^{2} b^{2}\left(\frac{3 a^{\prime} b^{\prime}}{2 a b}+\frac{a^{\prime \prime}}{2 a}+\frac{b^{\prime \prime}}{b}\right) S^{t r} \dot{t}+\frac{1}{2} r a^{\prime} S^{r \phi} \dot{\phi} \\
\dot{P}^{\phi} & +\frac{1}{r} P^{r} \dot{\phi}+\frac{1}{r} P^{\phi} \dot{r} \\
& =-\frac{a^{\prime}}{2 r a} S^{r \phi} \dot{r}+\frac{a b^{2}}{2 r}\left(a^{\prime}+2 a \frac{b^{\prime}}{b}\right) S^{t \phi} \dot{t}
\end{aligned}
$$

and the spin equations are given by

$$
\begin{aligned}
& \dot{S}^{t r}+\frac{b^{\prime}}{b} S^{t r} \dot{r}-r a S^{t \phi} \dot{\phi}=P^{t} \dot{r}-P^{r} \dot{t} \\
& \dot{S}^{r \phi}+\frac{1}{2} a b^{2}\left(a^{\prime}+2 a \frac{b^{\prime}}{b}\right) S^{t \phi} \dot{t}+\left(-\frac{a^{\prime}}{2 a}+\frac{1}{r}\right) \\
& S^{r \phi} \dot{r}=P^{r} \dot{\phi}-P^{\phi} \dot{r} \\
& \dot{S}^{t \phi}+\frac{1}{r} S^{t r} \dot{\phi}+\left(\frac{a^{\prime}}{2 a}+\frac{b^{\prime}}{b}+\frac{1}{r}\right) S^{t \phi} \dot{r} \\
& +\left(\frac{a^{\prime}}{2 a}+\frac{b^{\prime}}{b}\right) S^{r \phi} \dot{t}=P^{t} \dot{\phi}-P^{\phi} \dot{t} .
\end{aligned}
$$



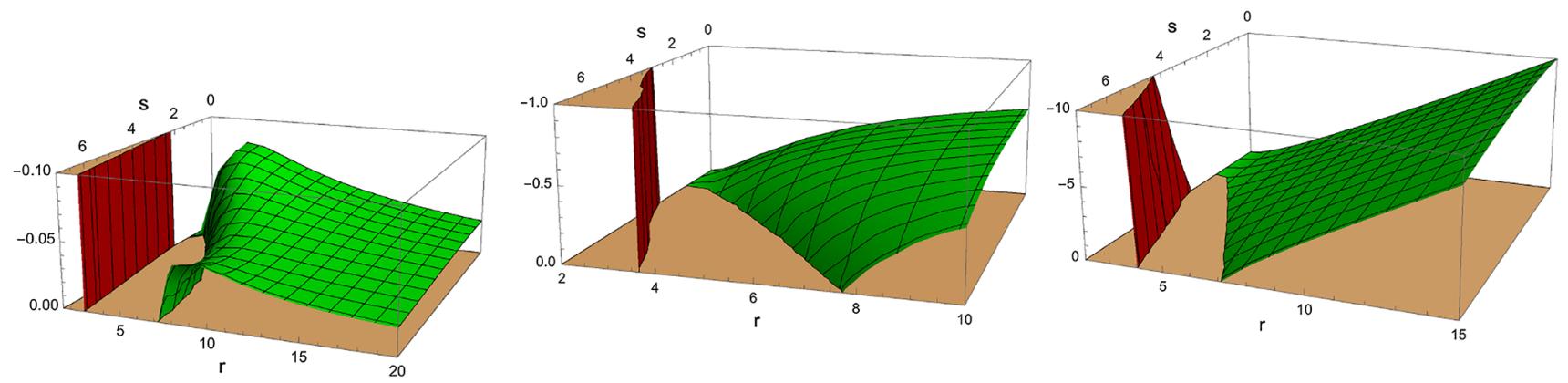

Fig. 10 The behavior of the velocity square $u_{\mu} u^{\mu} /\left(u^{t}\right)^{2}$ and $\Sigma$ as a function of $r$ and $s$, with $a 1=-2 M, M=1, b_{1}=1, E=1, j=-0.5$; left panel for $\sigma=0.1$, central panel for $\sigma=0$, and right panel for $\sigma=-0.1$

These equations imply

$$
\begin{aligned}
& \dot{r}=\frac{1-\frac{a^{\prime} s^{2}}{2 r}-\frac{a s^{2} b^{\prime}}{r b}}{1-\frac{a^{\prime} s^{2}}{2 r}} \frac{P^{r}}{P^{t}} \dot{t} \\
& \dot{\phi}=-\frac{-2+s^{2} a^{\prime \prime}+3 a^{\prime} \frac{b^{\prime}}{b} s^{2}+2 a \frac{b^{\prime \prime}}{b} s^{2}}{2\left(1-\frac{a^{\prime} s^{2}}{2 r}\right)} \frac{P^{\phi}}{P^{t}} \dot{t} .
\end{aligned}
$$

Using the above expressions we can evaluate the velocity square

$\frac{u_{\mu} u^{\mu}}{\left(u^{t}\right)^{2}}=-a(r) b(r)^{2}+\frac{1}{a(r)}\left(\frac{\dot{r}}{\dot{t}}\right)^{2}+r^{2}\left(\frac{\dot{\phi}}{\dot{t}}\right)^{2}$

In Fig. 10 we plot the behavior of $u_{\mu} u^{\mu} /\left(u^{t}\right)^{2}$ and $\Sigma$ as a function of $r$ and $s$ for some small values of $\sigma$. The green surface corresponds to the behavior of $u_{\mu} u^{\mu} /\left(u^{t}\right)^{2}$ and the red surface corresponds to the behavior of $\Sigma$. The intersection between the green surface and the $z=0$ horizontal plane is the limit where the trajectories change from timelike to spacelike character. The intersection between the red surface and the $z=0$ horizontal plane corresponds to the values for which CM energy diverges. We can observe that in order to reach a divergence in the energy of the center mass that the trajectory of the STOP has to pass from timelike to spacelike, which is similar to the collisions of spinning particles in the Schwarzschild background [54]. We recover the case for the Schwarzschild black hole when $\sigma=0$. See the central panel of Fig. 10.

\section{Conclusions}

In this paper we studied the motion of particles in the background of a DST black hole. We analyzed the motion of particles in the equatorial plane, and we recovered the classical result of GR for $\sigma=0$ and $r_{S}=2 M$ [73], recalling that $\sigma=0$ corresponds to $\beta_{n}=0$ due to its definition. A qualitative analysis of the effective potential for null geodesics shows that the behavior for radial photons is similar to those in a Schwarzschild's spacetime [73]. The same occurs for the motion of photons with angular momentum, when $-1 / 2<\sigma<1 / 4$, where unstable circular orbits depend on the coupling parameter $\sigma$. However, for $\sigma<-1 / 2$, all orbits are bounded, which does not occur in Schwarzschild's spacetime. The discrepancy between the theoretical value and the observational value of the deflection light was studied with respect to small deviations of Schwarzschild's spacetime. We have found via the geodesics formalism that the value of the coupling constant $\beta_{2}$ matches the theoretical result when the current observational constraints are $-8.97241 \times 10^{-6} \sqrt{3} \leq$ $\beta_{2} \leq 3.41708 \times 10^{-6} \sqrt{3}$.

Through the study for radial motion of massive particles, we obtain new geodesics: for $\sigma<0$ all orbits are bounded and for $0<\sigma<1 / 4$ an unstable equilibrium point $\left(r_{u}\right)$ and two critical trajectories approaching this point asymptotically with the same energy $E_{u}$ appear. For particles with $E>E_{u}$, the trajectories are unbounded. For $E<E_{u}$, we showed that they allow for a frontal scattering that is characterized for incoming particles from infinity to a radial distance of closest approach, and come back to infinity. With respect to the motion of particles with angular momentum and $\sigma<0$, all trajectories are bounded due to the potential at infinity. A Schwarzschild AdS black hole [68] and the classical GR orbits are also allowed. Interestingly, for $0<\sigma<1 / 4$, the spacetime has two unstable circular orbits and one stable circular orbit, and as the potential vanishes at infinity, not all the orbits are bounded. Also, there are planetary orbits, which allowed us to study the perihelion precession for Mercury and the discrepancy between the theoretical and observational values, for small deviations of Schwarzschild's spacetime. In this regard, we found that it is possible to attribute the discrepancy to a DST theory with a coupling constant $\beta_{2}=1.244 \times$ $10^{-9} \sqrt{3}$. Since the value of the coupling constant found for the perihelion precession is contained in the range obtained for the bending of light, these observables can be predicted by a unique DST theory according to observational data.

In consideration of the collision of spinning particles near the horizon and the possibility that the DST black hole acts as 
a particle accelerator, we showed that to reach a divergence in the center of mass energy, the trajectory of the STOP has to pass from timelike to spacelike. Thus, for small deviations of General Relativity, the behavior is similar to the one observed for collisions of spinning particles in the Schwarzschild background [54].

Acknowledgements We would like to thank the referee for valuable comments which helped us to improve the quality of this manuscript. We would also like to thank Thomas Mädler for his comments and suggestions. This work is partially supported by Comisión Nacional de Ciencias y Tecnología of Chile through FONDECYT Grant N 3170035 (A. Ö.), $\mathrm{N}^{\circ} 1170279$ (J. S.) and by the Dirección de Investigación y Desarrollo de la Universidad de La Serena (Y.V.). P. A. G. acknowledges the hospitality of the Universidad de La Serena where part of this work was undertaken. A. Ö. is grateful to the Institute for Advanced Study, Princeton, for its hospitality.

Data Availability Statement This manuscript has no associated data or the data will not be deposited. [Authors' comment: This is a theoretical paper without associated data.]

Open Access This article is distributed under the terms of the Creative Commons Attribution 4.0 International License (http://creativecomm ons.org/licenses/by/4.0/), which permits unrestricted use, distribution, and reproduction in any medium, provided you give appropriate credit to the original author(s) and the source, provide a link to the Creative Commons license, and indicate if changes were made. Funded by SCOAP ${ }^{3}$.

\section{References}

1. S. Deser, O. Sarioglu, B. Tekin, Gen. Relat. Gravit. 40, 1 (2008)

2. H. Weyl, Space-Time-Matter (Dover, New York, 1951)

3. T. Vetsov, arXiv:1806.05011 [gr-qc]

4. M. Olivares, J.R. Villanueva, Eur. Phys. J. C 73, 2659 (2013). arXiv:1311.4236 [gr-qc]

5. P.A. Gonzalez, M. Olivares, Y. Vasquez, Eur. Phys. J. C 75(10), 464 (2015). arXiv:1507.03610 [gr-qc]

6. M. Mathisson, Acta Phys. Pol. 6, 163 (1937)

7. A. Papapetrou, Proc. R. Soc. Lond. Ser. A 209, 248 (1951)

8. W. Tulczyjew, Acta Phys. Pol. 18, 393 (1959)

9. A.H. Taub, J. Math. Phys. 5, 112 (1964)

10. W.G. Dixon, Riv. Nuovo Cimento Soc. Ital. Fis. 34, 317 (1964)

11. E. Corinaldesi, A. Papapetrou, Proc. R. Soc. Lond. Ser. A 209, 259 (1951)

12. S. A. Hojman, PhD thesis, Princeton University (1975) (unpublished)

13. N. Zalaquett, S.A. Hojman, F.A. Asenjo, Class. Quantum Gravity 31, 085011 (2014). https://doi.org/10.1088/0264-9381/31/8/ 085011. arXiv:1308.4435 [gr-qc]

14. R. Hojman, S.A. Hojman, Phys. Rev. D 15, 2724 (1977)

15. M. Bañados, J. Silk, S.M. West, Phys. Rev. Lett. 103, 111102 (2009). arXiv:0909.0169 [hep-ph]

16. T. Piran, J. Shaham, J. Katz, Astrophys. J. 196, L107 (1975)

17. T. Piran, J. Shaham, Phys. Rev. D 16, 1615 (1977). https://doi.org/ 10.1103/PhysRevD.16.1615

18. T. Piran, J. Shaham, Astrophys. J. 214, 268 (1977)

19. A.A. Grib, Y.V. Pavlov, Int. J. Mod. Phys. D 20, 675 (2011). arXiv: 1008.3657 [gr-qc]

20. O.B. Zaslavskii, Phys. Rev. D 82, 083004 (2010). arXiv:1007.3678 [gr-qc]
21. O.B. Zaslavskii, JETP Lett. 92, 571 (2010)

22. O.B. Zaslavskii, Pisma. Zh. Eksp. Teor. Fiz. 92, 635 (2010). arXiv: 1007.4598 [gr-qc]

23. S. Gao, C. Zhong, Phys. Rev. D 84, 044006 (2011). arXiv: 1106.2852 [gr-qc]

24. Y. Li, J. Yang, Y.L. Li, S.W. Wei, Y.X. Liu, Class. Quantum Gravity 28, 225006 (2011). arXiv:1012.0748 [hep-th]

25. J.L. Said, K.Z. Adami, Phys. Rev. D 83, 104047 (2011). arXiv: 1105.2658 [gr-qc]

26. K. Lake, Phys. Rev. Lett. 104, 211102 (2010). [arXiv:1001.5463 [gr-qc]] (Erratum: [Phys. Rev. Lett. 104, 259903 (2010)])

27. A. Abdujabbarov, B. Ahmedov, B. Ahmedov, Phys. Rev. D 84, 044044 (2011). arXiv:1107.5389 [astro-ph.SR]

28. C. Zhong, S. Gao, JETP Lett. 94, 589 (2011). arXiv:1109.0772 [hep-th]

29. Y. Zhu, S. Fengwu, Y. Xiao Liu, Y. Jiang, Phys. Rev. D 84, 043006 (2011)

30. A. Abdujabbarov, N. Dadhich, B. Ahmedov, H. Eshkuvatov, Phys. Rev. D 88, 084036 (2013). arXiv:1310.4494 [gr-qc]

31. A. Galajinsky, Phys. Rev. D 88, 027505 (2013). https://doi.org/10. 1103/PhysRevD.88.027505. arXiv:1301.1159 [gr-qc]

32. S.R. Shaymatov, B.J. Ahmedov, A.A. Abdujabbarov, Phys. Rev. D 88(2), $024016(2013)$

33. J. Sadeghi, B. Pourhassan, H. Farahani, Commun. Theor. Phys. 62(3), 358 (2014). arXiv:1310.7142 [hep-th]

34. S. Fernando, Gen. Relativ. Gravit. 46, 1634 (2014). arXiv:1311.1455 [gr-qc]

35. J. Yang, Y.L. Li, Y. Li, S.W. Wei, Y.X. Liu, Adv. High Energy Phys. 2014, 204016 (2014). arXiv:1202.4159 [hep-th]

36. S.G. Ghosh, P. Sheoran, M. Amir, Phys. Rev. D 90(10), 103006 (2014). arXiv:1410.5588 [gr-qc]

37. P. Pradhan, Astropart. Phys. 62, 217 (2015). arXiv:1407.0877 [grqc]

38. P. Pradhan,. arXiv:1402.2748 [gr-qc]

39. U. Debnath,. arXiv:1508.02385 [gr-qc]

40. S.G. Ghosh, M. Amir, Eur. Phys. J. C 75(11), 553 (2015). arXiv: 1506.04382 [gr-qc]

41. M. Amir, S.G. Ghosh, JHEP 1507, 015 (2015). arXiv:1503.08553 [gr-qc]

42. B. Pourhassan, U. Debnath, arXiv:1506.03443 [gr-qc]

43. M. Guo, S. Gao, Phys. Rev. D 93(8), 084025 (2016). arXiv: 1602.08679 [gr-qc]

44. Y.P. Zhang, B.M. Gu, S.W. Wei, J. Yang, Y.X. Liu, Phys. Rev. D 94(12), 124017 (2016). arXiv:1608.08705 [gr-qc]

45. O.B. Zaslavskii, EPL 114(3), 30003 (2016). arXiv:1603.09353 [grqc]

46. O.B. Zaslavskii, Int. J. Mod. Phys. D 26(10), 1750108 (2017). arXiv: 1602.08779 [gr-qc]

47. N. Tsukamoto, K. Ogasawara, Y. Gong, Phys. Rev. D 96(2), 024042 (2017). arXiv:1705.10477 [gr-qc]

48. S. Fernando, Mod. Phys. Lett. A 32, 1750074 (2017). arXiv: 1703.00373 [gr-qc]

49. M. Halilsoy, A. Ovgun, Can. J. Phys. 95(11), 1037 (2017). arXiv:1507.00633 [gr-qc]

50. M. Halilsoy, A. Ovgun, Adv. High Energy Phys. 2017, 4383617 (2017). arXiv:1504.03840 [gr-qc]

51. R. Becar, P.A. Gonzalez, Y. Vasquez, Eur. Phys. J. C 78(4), 335 (2018). arXiv:1712.00868 [gr-qc]

52. P.A. Gonzalez, M. Olivares, E. Papantonopoulos, Y. Vasquez, Phys. Rev. D 97(6), 064034 (2018). arXiv:1802.01760 [gr-qc]

53. F. Atamurotov, B. Ahmedov, S. Shaymatov, Astrophys. Space Sci. 347, 277 (2013)

54. C. Armaza, M. Bañados, B. Koch, Class. Quantum Gravity 33(10), 105014 (2016). arXiv: 1510.01223 [gr-qc]

55. L.F. Abbott, S. Deser, Phys. Lett. 116B, 259 (1982) 
56. L.F. Abbott, S. Deser, Nucl. Phys. B 195, 76 (1982). https://doi. org/10.1016/0550-3213(82)90049-9

57. S. Deser, B. Tekin, Phys. Rev. D 67, 084009 (2003). arXiv:hep-th/0212292

58. S. Deser, B. Tekin, Phys. Rev. Lett. 89, 101101 (2002). aXiv:hepth/0205318

59. W. Kim, S. Kulkarni and S. H. Yi, Phys. Rev. Lett. 111, no. 8, 081101 (2013 arXiv:1306.2138 [hep-th] () Erratum: [Phys. Rev. Lett. 112, no. 7, 079902 (2014)] )

60. H. Adami, M.R. Setare, T.C. Sisman, B. Tekin,. arXiv:1710.07252 [hep-th]

61. V. Perlick, Living Rev. Relativ. 7, 9 (2004)

62. V. Bozza, Gen. Relativ. Gravit. 42, 2269 (2010)

63. Ivan Zh Stefanov, Stoytcho S Yazadjiev, Galin G Gyulchev, Phys. Rev. Lett 104, 251103 (2010)

64. M. Bartelmann, P. Schneider, Phys. Rep. 340, 291 (2001)

65. N. Straumann, General Relativity and Relativistic Astrophysics (Springer-Verlag, Berlin Heidelberg New York Tokio, 1984)
66. J.R. Villanueva, M. Olivares, Eur. Phys. J. C 75(11), 562 (2015). arXiv: 1510.08340 [gr-qc]

67. J. Ramos-Caro, J.F. Pedraza, P.S. Letelier, Mon. Not. R. Astron. Soc. 414, 3105 (2011). arXiv:1103.4616 [astro-ph.EP]

68. N. Cruz, M. Olivares, J.R. Villanueva, Class. Quantum Gravity 22, 1167 (2005). arXiv:gr-qc/0408016

69. S. Cornbleet, Am. J. Phys. 61, 650 (1993)

70. R. Wald, General Relativity (University of Chicago Press, Chicago, 1984)

71. B. Shutz, A First Course in General Relativity (Cambridge University Press, Cambridge, 1990)

72. S.S. Shapiro, J.L. Davis, D.E. Lebach, J.S. Gregory, Phys. Rev. Lett. 92, 121101 (2004)

73. S. Chandrasekhar, The Mathematical Theory of Black Holes (Oxford University Press, New York, 1983) 